\title{
Screening for diabetic nephropathy at diabetes clinic in Sudan
}

\author{
Abdul-Azim Ali Abdul-Azim ${ }^{1}$, Awad Ali M. Alawad ${ }^{2} *$, Faisal H. Younis ${ }^{2}$ \\ ${ }^{1}$ MBBS, Faculty of Medicine, University of Medical Sciences and Technology, Sudan \\ ${ }^{2}$ Assistant Professor, Faculty of Medicine, University of Medical Sciences and Technology, Sudan \\ *Corresponding author E-mail: awadali82@hotmail.com
}

\begin{abstract}
Background: Diabetes mellitus and its complications are one of the major health problems in Sudan. Only few patients had been screened before their complications appeared. As a result of this, many patients have not been discovered and ended with late stages of the disease, and are now showing evidence of diabetic nephropathy.

Objective: The main aim of this study is at discovering the factors in terms of patients' knowledge that are leading to the defect in diabetic nephropathy screening at one of the diabetes clinics.

Methods: This cross-sectional hospital-based descriptive study was conducted at University charity teaching hospital affiliated to University of Medical Sciences and Technology (UMST) and covered 56 patients diagnosed with Diabetes Mellitus during the period from August to October 2013. Information was obtained by direct interviewing of patients using a questionnaire, and filled in the form of an interview.

Results: A total of 56 patients were included, their mean age group was $60-69$ and of them $51.8 \%$ were males. Regarding awareness about the symptoms of diabetic nephropathy, the most known symptom was fatigue, known by 27 (48.2\%). Out of all the participants 55 $(98.2 \%)$ reported checking their blood pressure regularly. A total of $50(89.2 \%)$ believed that smoking had a harmful effect on their diabetes and $12(21.4 \%)$ participants smoke cigarettes. Regarding exercise $(54,96.4 \%)$ believed that exercise is helpful in decreasing the incidence of diabetic nephropathy, however only $16(28.6 \%)$ participants reported exercising regularly. A total of 51 (91\%) have heard about the screening for diabetic nephropathy yet only $46(82.1 \%)$ participant had their screening done regularly.

Conclusion: Majority of participants had an understanding of the factors and knowledge on the signs of diabetic nephropathy, yet not all have evaded the risk factors. Raising awareness is essential but providing facilities to ensure its compliance is required.
\end{abstract}

Keywords: Diabetes Mellitus; Nephropathy.

\section{Introduction}

About 14 million individuals were estimated to have diabetes mellitus (DM) in Africa (Abdelgadir et al. 2006), a figure expected to rise to 28 million by the year 2030 . Prior reports showed that DM was associated with poor glycemic control, high prevalence of complications, low quality of life and increased morbidity (MakkiAwouda et al. 2013).

Chronic kidney disease is at least 3-4 times more frequent in Africa than in developed countries (Elrayah-Eliadarous et al. 2010). The prevalence of diabetic nephropathy is estimated to be $14-16 \%$ in South Africa, 23.8\% in Zambia, 12.4\% in Egypt, 9\% in Sudan, and $6.1 \%$ in Ethiopia. The current dialysis treatment rate ranges from 70 per million populations in South Africa to $<20 \mathrm{pmp}$ in the most of sub-Saharan Africa (Ahmed \& Abdelrhan 2006).

Diabetic nephropathy (DN) a known complication of DM remains a major cause of end-stage renal disease (ESRD) worldwide, as well as major causes of death in Sudan. The overall prevalence of chronic kidney disease (CKD) in the Sudan was estimated to range from $7.7 \%$ to $11 \%$ of the population; with $13.3 \%$ of CKD patients being diabetics (Abdelgadir et al. 2006). Diabetic nephropathy was reported as the third most common cause of patients being on dialysis therapy in Sudan, i.e. accounting for $10.4 \%$ of all cases. In our survey, as well as in previous reports from the Sudan, it is alarming to note that a considerable proportion of patients with diabetes remain far from achieving their glycemic goals. This again raises the issue of having a multidisciplinary set-up for implementing the international guidelines of screening and management of diabetes; as well as early detection of its complications.

\section{Methods}

This is a cross-sectional study that relied on conducting face-toface interviews using a structured questionnaire with a random sample of patients admitted to University charity teaching hospital, University of Medical Sciences and Technology, Khartoum, Sudan. This study was carried out during the period from beginning of August to end of October 2013.

The questionnaire was designed specifically for the purpose of the study having consulted the medical literature of similar studies. The questionnaire was piloted on a sample of 15 patients to ensure face validity and clarity. Minor changes were made; namely rewording two questions. Those interviews were excluded from the analysis. The questionnaire contained 18 items, including demographic and socio-economic data. Face-to-face interviews using the questionnaire were conducted. Interviews took place at diabetes clinic.

Data were entered into a Microsoft Excel spreadsheet and the statistical analysis was conducted using SPSS (Version 17). Statistical analysis was carried out using descriptive and analytical sta- 
tistics. Simple frequencies and cross tabulation were done. Chi square test was used for proportions. Stratification for the patient's sex and educational level was done when relevant. $P$ value of less than 0.05 was considered statistically significant.

The consent of the patients was obtained. A full explanation of the purposes and nature of the study was conveyed to them. The potential participants were clearly assured that their participation in this study is voluntary any data obtained would be treated confidentially and for the purpose of the research only.

\section{Results}

The total number of participants in the study was 56 . Of these 29 $(51.8 \%)$ are males whereas $27(48.2 \%)$ are females. The most common age group among the participants was 60-69 which accounted for $20(35.7 \%)$ of the participants whereas $13(23.2 \%)$ fall into the age group 70-79, with a mean age of 58.6 for all patients; 57.7 and 59.6 for males and females respectively.

Regarding treatment regimen taken, it was noticed that $23(41.1 \%)$ of participants were taking insulin and the same number, 23 $(41.1 \%)$, of participants were taking oral hypoglycemic agents, whereas $7(12.5 \%)$ were on diet and exercise and $3(5.3 \%)$ participants were not on any treatment. Of the participants on treatment, $53(94.6 \%)$ were taking their medication regularly, with females being slightly more compliant $(26,96.3 \%)$ compared to males $(27$, $93.1 \%)$. Fig. 1 shows the treatment regimen taken by the participants.

Regarding awareness about the symptoms of diabetic nephropathy, $27(48.2 \%)$ participants knew that fatigue is a symptom, 25 (44.6\%) participants knew urine changes is a symptom, 19 $(33.9 \%)$ participants knew that body swelling is a symptom, 16 $(28.6 \%)$ participants knew that nausea and vomiting is a symptom, $13(23.2 \%)$ participants knew that loss of appetite is a symptom, $12(21.4 \%)$ participants knew that weight gain is a symptom, 9 (16.1\%) participants did not know about any symptom of diabetic nephropathy, $6(10.7 \%)$ participants knew that abdominal pain is a symptom and $5(8.9 \%)$ participants knew that headache is a symptom of diabetic nephropathy. Fig. 2 shows the symptoms of diabetic nephropathy known by the participants.

Out of all the participants, 54 (96.4\%) of them knew that the regularity in screening and taking the treatment would help in preventing the appearance of diabetic nephropathy. Out of all the participants, $43(76.8 \%)$ knew that time is a factor associated with diabetic nephropathy. All the participants, $56(100 \%)$ agree that checking the blood sugar level is important to help prevent complications of diabetes, and even though 55 (98.2\%) reported checking their blood pressure regularly, only 47 participants $(83.9 \%)$ think that checking the blood pressure is also important. Out of all the participants, $50(89.2 \%)$ believed that smoking had a harmful effect on their diabetes and $12(21.4 \%)$ participants smoke cigarettes. Regarding exercise, almost all the participants (54, 96.4\%) believed that exercise is helpful in decreasing the incidence of diabetic nephropathy, however only 16 (28.6\%) participants reported exercising regularly. Fig. 3 shows the factors known by the participants to be associated with the development of diabetic nephropathy.

A total of $51(91 \%)$ have heard about the screening for diabetic nephropathy, with females being more $(25,92.6 \%)$ compared to males $(25,86.2 \%)$. Out of all the participants, $47(83.9 \%)$ believed that screening is beneficial in preventing diabetic nephropathy, with females being more $(24,88.9 \%)$ compared to males $(23$, $79.3 \%$ ). The participants differed about how screening protects from diabetic nephropathy; $23(41.1 \%)$ participants believed that screening protects against development of diabetic nephropathy itself, while $26(46.4 \%)$ participants believed it helps by early discovery of the diabetic nephropathy, $3(5.3 \%)$ participants believed it helps by decreasing the damage done by this complication and $8(14.3 \%)$ participants thought it is of no benefit. A total of $46(82.1 \%)$ participant had their screening done regularly with females being more $(24,88.9 \%)$ compared to males $(22,75.9 \%)$.

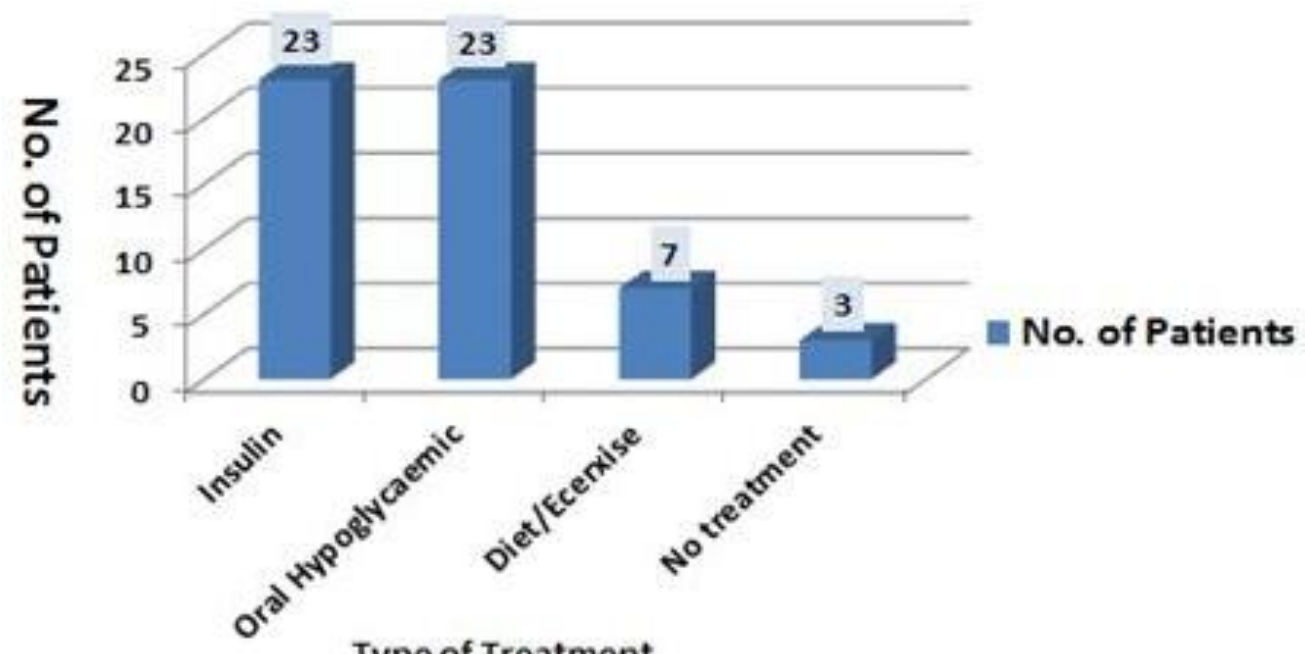

Type of Treatment

Fig. 1: Shows the Treatment Regimen Taken by the Participants 


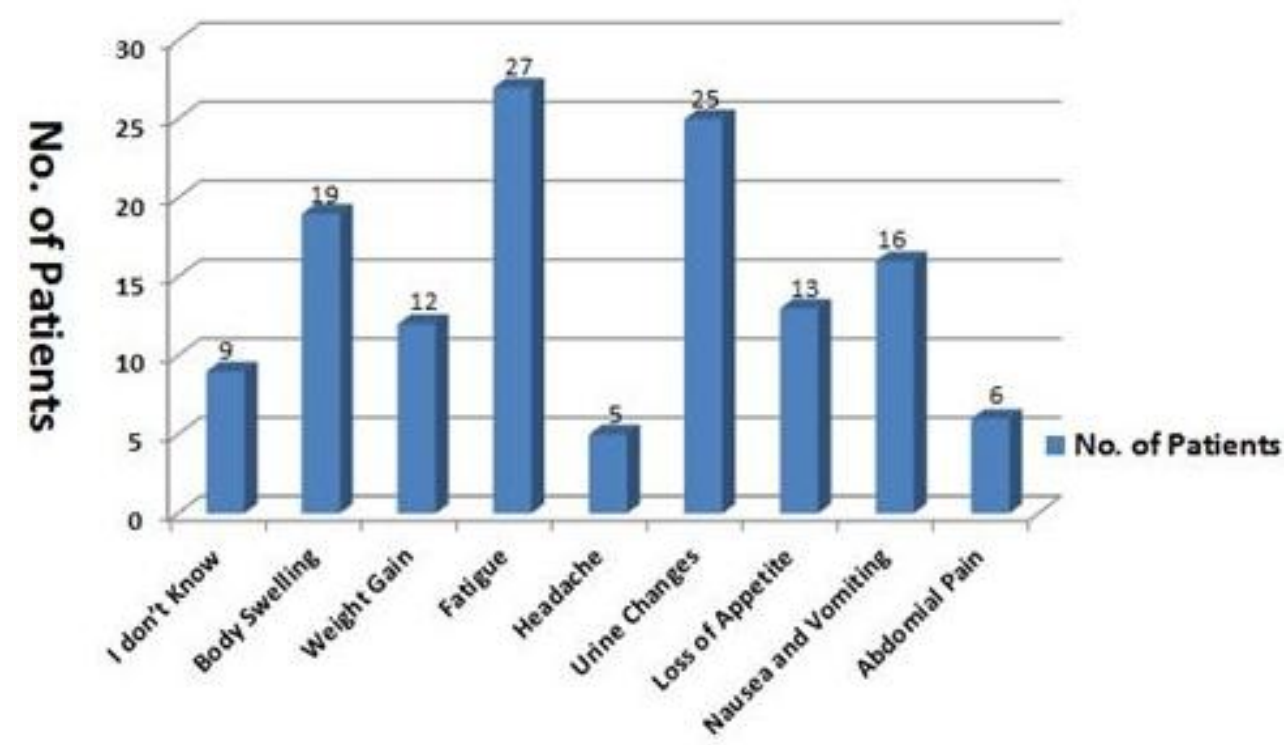

Svmptoms

Fig. 2: Shows the Symptoms of Diabetic Nephropathy Known by the Participants

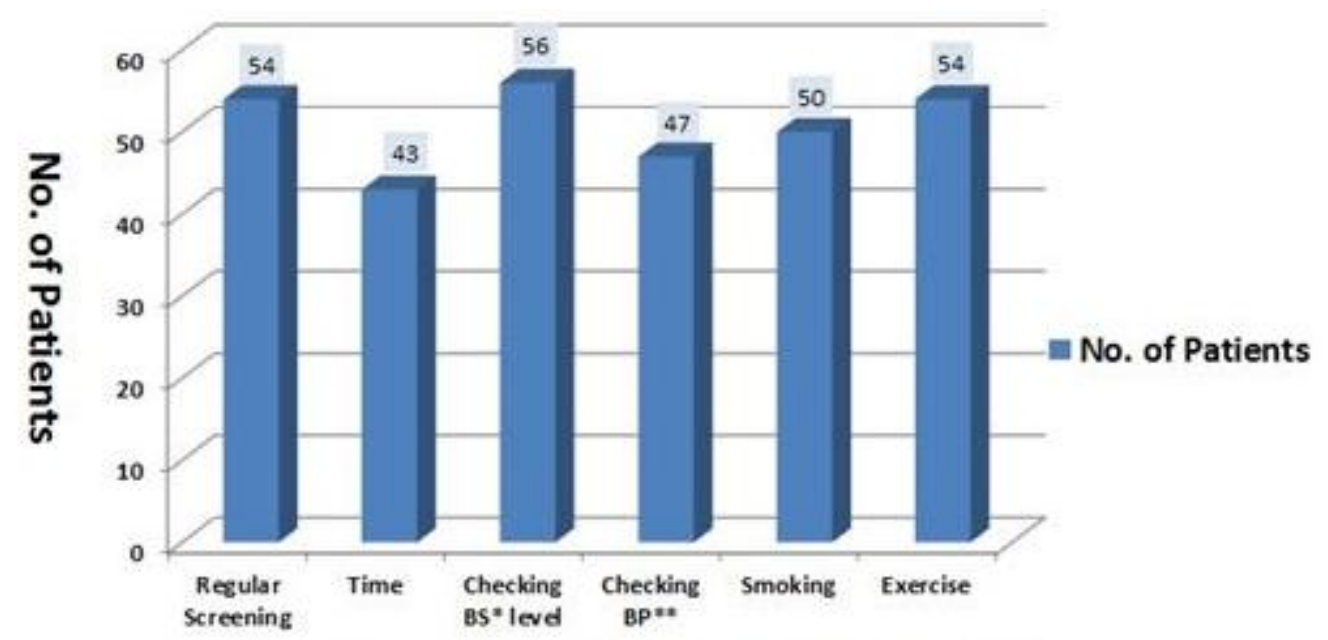

Factors affectig development of Diabetic Nephropathy

Fig. 3: Shows the Factors Known by the Participants to Be Associated with the Development of Diabetic Nephropathy (* Blood Sugar ** Blood Pressure)

\section{Discussion}

Health services in the form of multidisciplinary care centers remain scarce in Sudan, with the personnel trained in diabetes care being very few or almost non-existent. Illiteracy, lack of health education, poverty and dominance of wrong beliefs adversely affect the management of diabetes among the Sudanese patients (Abdelgadir et al. 2002). Prior reports estimated the overall prevalence of long-term complications in Sudanese adults, with a median duration of diabetes of 9 years, as $67 \%$. Among these the prevalence of retinopathy, neuropathy, cardiovascular disease, nephropathy, peripheral vascular disease, and cerebrovascular accidents were $43 \%, 37 \%, 28 \%, 22 \%, 10 \%$ and $5.5 \%$, respectively (Rim et al. 2013).

In this study the results for treatment regimen taken showed that $23(41.1 \%)$, of participants were taking oral hypoglycemic agents, which is different from Shigidi research conducted in Khartoum State, Sudan; which showed that $57.9 \%$ were on oral hypoglycemic agents (Ahmed \& Abdelrhan 2006). Regarding the benefits of exercise, almost all the participants $(54,96.4 \%)$ believed that exercise is helpful in decreasing the incidence of diabetic nephropathy, however only 16 (28.6\%) participants reported exercising regularly. This was consistent with Shigidi research conducted in
Khartoum State, Sudan; which showed that $25 \%$ of the participants were on regular exercise. This is probably due to the fact that both researches were done in Khartoum.

Regarding smoking, it was found that $78.6 \%$ of the participants where nonsmokers. This is closely similar to Shigidi research conducted in Khartoum State, Sudan; which showed that $69.7 \%$ are non-smokers. Regarding the effects of smoking, 50 (89.2\%) of the participants believed that smoking had a harmful effect on their diabetes, this is more than Mumtaz study (Kraft et al. 1999) which showed $73.4 \%$. Regarding screening, it was found that $17.9 \%$ of the participants didn't do regular follow up; this is substantially lower than found in Chaudhary study which showed $77 \%$ were not having regular checkup (Elbagir et al. 1998). This can be due to the fact that most of the participants in the study were from the dialysis center where tests were done on each admission.

\section{Conclusions}

Majority of participants had an understanding of the factors and knowledge on the signs of diabetic nephropathy, yet not all have evaded the risk factors. This was evident in the study since although the participants had knowledge on the benefits and risk 
factors, a much lower percentage was found to act accordingly. Raising awareness is essential but providing facilities to ensure its compliance is required.

\section{References}

[1] Abdelgadir M, Elbagir M, Eltom M \& Berne C (2006): The influence of glucose self-monitoring on glycaemic control in patients with diabetes mellitus in Sudan. Diabetes Res Clin Pract 74, 90-94. http://dx.doi.org/10.1016/j.diabres.2006.03.003.

[2] Abdelgadir M, Elbagir M, Eltom M, Berne C \& Ahren B (2002): Reduced leptin concentrations in subjects with type 2 diabetes mellitus in Sudan. Metabolism 51, 304-306. http://dx.doi.org/10.1053/meta.2002.30504.

[3] Ahmed ME \& Abdelrhan SH (2006): The role of medical students in patient education to promote home management of diabetes mellitus in wad medani town, Sudan 2003. J Family Community Med 13, 4146.

[4] Elbagir MN, Eltom MA, Elmahadi EM, Kadam IM \& Berne C (1998): A high prevalence of diabetes mellitus and impaired glucose tolerance in the Danagla community in northern Sudan. Diabet Med 15, 164 $169 . \quad$ http://dx.doi.org/10.1002/(SICI)10969136(199802)15:2<164::AID-DIA536>3.0.CO;2-A

[5] Elrayah-Eliadarous H, Yassin K, Eltom M, Abdelrahman S, Wahlstrom R \& Ostenson CG (2010): Direct costs for care and glycaemic control in patients with type 2 diabetes in Sudan. Exp Clin Endocrinol Diabetes 118, 220-225. http://dx.doi.org/10.1055/s-00291246216.

[6] Kraft SK, Lazaridis EN, Qiu C, Clark CM, Jr. \& Marrero DG (1999) Screening and treatment of diabetic nephropathy by primary care physicians. J Gen Intern Med 14, 88-97. http://dx.doi.org/10.1046/j.15251497.1999.00292.x.

[7] MakkiAwouda FO, Elmukashfi TA \& Hag Al-Tom SA (2013): Designing an educational and training program for diabetes health educators at Diabetic Health Centers, Khartoum State, Sudan; 2007-2010. Glob J Health Sci 5, 207-211.

[8] Rim TH, Byun IH, Kim HS, Lee SY \& Yoon JS (2013): Factors associated with diabetic retinopathy and nephropathy screening in Korea: the Third and Fourth Korea National Health and Nutrition Examination Survey (KNHANES III and IV). J Korean Med Sci 28, 814-820. http://dx.doi.org/10.3346/jkms.2013.28.6.814. 